\title{
A Study of Analysis of Critical Values at Emergency Biochemistry Laboratory in a Tertiary Care Hospital in India
}

\author{
Namrata Bhutani ${ }^{1}$, Neha Bhutani ${ }^{2}$ \\ ${ }^{1}$ Department of Biochemistry, Vardhaman Mahavir Medical College and Safdarjung Hospital, \\ New Delhi, India. ${ }^{2}$ ESIC Dental College, Rohini, New Delhi, India.
}

\section{ABSTRACT}

\section{BACKGROUND}

One of the important functions of a clinical biochemistry laboratory is clear, accurate, and rapid communication of a critical value to clinicians ${ }^{1}$. Critical value reporting is one of the requirements for laboratory accreditation. This study aimed to analyse the critical values data at a tertiary care hospital in New Delhi, India in the emergency laboratory setting and compare it with the available data from previous studies.

\section{METHODS}

This is a retrospective study of emergency biochemistry samples done over a period of two months from July 2019 to September 2019. The critical values data of several biochemical parameters generated from the testing of samples on Beckman Coulter Olympus AU 480 during three months period were analysed. Statistical analysis was conducted using Microsoft Excel 2016 program.

\section{RESULTS}

183056 tests were analysed, and 11875 critical alerts were generated the incidence of which varied from $0.8 \%$ for glucose (children) to $10.6 \%$ for serum potassium in children. Frequency of critical values for serum potassium is highest followed by serum sodium. Also, critical values for serum potassium in paediatric patients is higher than in adults. It can be stated that there is a need to study of frequency of critical values in emergency as well as routine laboratories to identify the parameters with maximum critical values and also recognize the underlying causes for the same and take suitable measures.

\section{CONCLUSIONS}

The frequency of critical value for serum potassium, is highest followed by serum sodium, in case of samples received from adult patients. Also, frequency of critical values for serum potassium in paediatric patients is even higher than adults. These values show that these critical biochemical values can be detrimental to these patients and timely notification to the clinician is very important so that necessary actions could be taken to correct them. Further studies of critical values in emergency labs is required to observe trends and identify the underlying causes and suggest suitable remedial measures if any.

\section{KEY WORDS}

Critical Value, Biochemistry, Emergency Laboratory, Pre-Analytical Errors
Corresponding Author: Dr. Namrata Bhutani, D-162, Tagore Garden Extension, New Delhi-110027, India. E-mail:namrata.bhutani@gmail.com

DOI: $10.14260 / \mathrm{jemds} / 2020 / 206$

Financial or Other Competing Interests: None.

How to Cite This Article:

Bhutani N, Bhutani N. A study of analysis of critical values at emergency biochemistry laboratory in a tertiary care hospital in India. J. Evolution Med. Dent. Sci. 2020;9(12):957-960, DOI: 10.14260/jemds/2020/206

Submission 31-12-2019, Peer Review 27-02-2020,

Acceptance 05-03-2020,

Published 23-03-2020.

\section{(i)}




\section{BACKGROUND}

A critical value can be defined as "a laboratory test result that represents a pathophysiologic state at such variance with normal as to be life threatening unless something is done promptly and for which some corrective action could be taken". One of the important functions of a clinical biochemistry laboratory is clear, accurate, and rapid communication of a critical value to clinicians. ${ }^{1}$ Critical value reporting is one of the requirements for laboratory accreditation. It is important to instantly communicate critical values for faster diagnostic process and quick change in patient management. Thus, informing of critical values is an important task; but, there are no clear guidelines for identifying who should be responsible for receiving the critical values notification (consultant, senior or junior resident doctor, intern, nurse, etc.). This notification should be checked, recorded, informed in the respective wards as well as confirmed that the health-care provider has accepted responsibility for follow up. Thus, the critical value notification procedure mentioned must be carefully considered by clinical laboratories, as it directly impacts patient safety, efficiency and quality of clinical care. Moreover, the current literature indicates that there is no guidelines on the parameters which are more likely to result in critical values, nor on the critical value limits (both upper and lower). ${ }^{2}$ Thus, it is imminent that a consensus must be reached among laboratory specialists on the list of parameters and the critical value limits of each of them, which should be established and followed by each laboratory. ${ }^{2}$

Among the many published guidelines for the reporting of critical results, one is issued by the British Royal College of Pathologists. Additionally, independent lists are required for different study populations, as critical values vary among neonatal, paediatric and adult care patients. Defining and mandating a universal set of thresholds for tests, however, is a difficult task given the scarcity of outcomes based data on critical value thresholds. ${ }^{2}$ Critical value lists that are too inclusive (or that have critical value thresholds that require excessive notification) place an unnecessary burden on laboratory staff, annoy clinicians and create a negative attitude toward important laboratory services. . $^{3,4}$

On the other hand, lists that are too exclusive (or with thresholds that are too high or low) might not prevent adverse clinical outcomes, as a delay in the recognition of lifethreatening laboratory results by clinicians can be disastrous $^{5}$. To establish or modify critical values, it is important to compare with previously published lists, practice parameters, and consensus documents because these sources have been refined with the benefit of time, institutional comparison, and clinical performance.6,7 Most reports have analysed only a few analyses for short periods or have reviewed a small number of critical values in different institutions. 8

In this present study, we aimed to analyse the critical values data at a tertiary care hospital in New Delhi, India in the emergency laboratory setting, for a period of two months and compare the frequencies for different parameters with the available data from previous studies.

\section{METHODS}

The current retrospective study was conducted at the Biochemistry laboratory of new emergency building of Safdarjung Hospital, New Delhi, India. This laboratory receives blood samples from all the emergency wards as well as non-routine samples for inpatient patients from rest of the wards of the hospital (including medicine, surgery, gynaecology, paediatrics and all other wards in the hospital). The biochemistry parameters that are done on blood samples in emergency laboratory include glucose, urea, creatinine, total bilirubin, direct bilirubin, Alkaline Phosphatase (ALP), Alanine Transaminase (ALT), Aspartate transaminase (AST), amylase, sodium, potassium, chloride, creatine kinase (CK), creatine kinase-MB (CK-MB) and lipase. All the samples which are received in the laboratory are run on Beckman Coulter Olympus AU 480 For the purpose of this study, data was collected from entry registers and rejected samples registers, which are maintained in the laboratory. The emergency biochemistry samples were evaluated retrospectively over a period of two months from July 2019 to September 2019 and total of 183056 tests were studied in this study. The parameters which were chosen by the lab in charge to be notified for critical values include glucose, serum creatinine, blood urea, serum sodium, potassium, chloride, CSF glucose and CK-MB in adults and total bilirubin, serum potassium, creatinine, glucose and CSF glucose in children and newborn patients. The critical value result is checked for analytical reliability, then after checking and ruling out the pre- analytical errors as a potential cause of critical values, the sample is run again. Once the result is validated, the result is considered as a true critical value and it is notified by the doctor on duty telephonically to the clinicians in the respective wards and the same is recorded in a register. This is the protocol followed in this laboratory for critical value notification. The list of critical values and their biological limits used for this study are presented in table 1 . These limits were designed based on available literature ${ }^{9,10}$ and by consultation of the clinicians of our hospital.

\begin{tabular}{|c|c|c|}
\hline Test & Lower Limit & Upper Limit \\
\hline Adults & & \\
\hline Glucose & $40 \mathrm{mg} / \mathrm{dl}$ & $450 \mathrm{mg} / \mathrm{dl}$ \\
\hline Sodium & $120 \mathrm{meq} / \mathrm{l}$ & $160 \mathrm{meq} / \mathrm{l}$ \\
\hline Potassium & $2.8 \mathrm{meq} / \mathrm{l}$ & $6.2 \mathrm{meq} / \mathrm{l}$ \\
\hline S. Chloride & $80 \mathrm{meq} / \mathrm{l}$ & $120 \mathrm{meq} / \mathrm{l}$ \\
\hline Urea & & $171 \mathrm{mg} / \mathrm{dl}$ \\
\hline Creatinine & & $5 \mathrm{mg} / \mathrm{dl}$ \\
\hline CSF Glucose & $40 \mathrm{mg} / \mathrm{dl}$ & $100 \mathrm{mg} / \mathrm{dl}$ \\
\hline CK-MB & & $25 \mathrm{U} / \mathrm{L}$ \\
\hline Newborn & & $>15 \mathrm{mg} / \mathrm{dl}$ \\
\hline T. Bilirubin (Newborn ) & & 7.8 \\
\hline S. Potassium ( Newborn ) & $2.8 \mathrm{meq} / \mathrm{l}$ & 3.8 \\
\hline Creatinine & & $445 \mathrm{mg} / \mathrm{dl}$ \\
\hline Glucose (Children ) & $46 \mathrm{mg} / \mathrm{dl}$ & $325 \mathrm{mg} / \mathrm{dl}$ \\
\hline Glucose (Newborn ) & $30 \mathrm{mg} / \mathrm{dl}$ & $188 \mathrm{mg} / \mathrm{dl}$ \\
\hline CSF Glucose (Children ) & $31 \mathrm{mg} / \mathrm{dl}$ & \\
\hline CSF Protein (Children ) & & \multicolumn{2}{c|}{} \\
\hline \multicolumn{2}{|c|}{ Table 1. Critical Value Limits Range in Our Laboratory } \\
\hline \multicolumn{2}{|c|}{} \\
\hline
\end{tabular}

\section{Statistical Analysis}

Statistical analysis was conducted using Microsoft Excel 2016 program. Calculations of critical values of biochemistry lab was presented as number and percentage. 


\section{RESULTS}

As explained above in methodology, the emergency biochemistry samples were evaluated retrospectively over a period of three months from July 2019 to September 2019. For the purpose of this study, a total of 183056 tests were analysed during the study period and 11875 critical alerts were generated by the Olympus AU 480 analyser in total during this period. Thus, it can be observed that number of critical values comprised of $6.5 \%$ of total tests done. Moreover, both lower critical values and higher critical values of all the eligible parameters were recorded separately and total percentage of critical values for each parameter were recorded as explained in Table 2 below. It can be seen in this table that in samples from adult patients, $7.1 \%$ tests done for glucose were in critical value range. In the case of urea, 3.4\% tests were in critical value range. Ical value notification in adult patients. Thus, urea was observed to have the minimum frequency for crit Moreover, out of 33509 tests done for creatinine, 2010 critical value tests were reported, which is $5.9 \%$ of the total number of creatinine tests. In the case of electrolytes, 2933 critical values were reported out of a total of 35331 tests done for potassium (K+). This is $8.3 \%$ of the total potassium tests done in adults, and is having the maximum frequency among the critical values reported were adult patients. Also, in the case of sodium $(\mathrm{Na}+)$ this frequency was observed to be $7.2 \%$. The frequency of critical value notification was observed to be $4.6 \%$ and $5.5 \%$ in case of CSF Glucose and CK-MB as can be seen in table 2 .

\begin{tabular}{|c|c|c|c|c|c|}
\hline Test & Total & Critical Values & Lower & Upper & $(\%)$ \\
\hline \multicolumn{6}{|c|}{ Adults } \\
\hline Glucose & 7312 & 525 & 95 & 430 & $7.1 \%$ \\
\hline Sodium & 35331 & 2566 & 1078 & 1488 & $7.2 \%$ \\
\hline Potassium & 35331 & 2933 & 1425 & 1508 & $8.3 \%$ \\
\hline S. Chloride & 35 & 2 & 2 & 0 & $5.7 \%$ \\
\hline Urea & 33512 & 1145 & & & $3.4 \%$ \\
\hline Creatinine & 33509 & 2010 & & & $5.9 \%$ \\
\hline CSF Glucose & 1018 & 47 & 27 & 20 & $4.6 \%$ \\
\hline $\mathrm{CK}-\mathrm{MB}$ & 108 & 6 & & & $5.5 \%$ \\
\hline \multicolumn{6}{|c|}{ Newborn } \\
\hline T. Bilirubin (Newborn) & 10345 & 1080 & & & $10.4 \%$ \\
\hline S. Potassium (Newborn) & 11234 & 1195 & & & $10.6 \%$ \\
\hline Creatinine & 10976 & 321 & & & $2.9 \%$ \\
\hline Glucose (Children) & 1831 & 15 & 10 & 5 & $0.8 \%$ \\
\hline Glucose (Newborn) & 2023 & 18 & 7 & 11 & $0.9 \%$ \\
\hline CSF glucose (Children) & 254 & 5 & 2 & 3 & $1.9 \%$ \\
\hline CSF protein (Children) & 237 & 7 & 4 & 3 & $2.9 \%$ \\
\hline Total & 183056 & 11875 & & & $6.5 \%$ \\
\hline \multicolumn{6}{|c|}{ Table 2. Critical Values of Different Parameters Tested } \\
\hline
\end{tabular}

In case of samples received from newborn patients from the paediatric wards, out of total 10345 samples analysed for total bilirubin, 1080 samples were reported to be in the critical range. This comprises of $10.4 \%$ of total bilirubin samples in newborns. Also, in the case of direct bilirubin, out of 11234 samples analysed, 1195 samples were in the critical value range, thus leading to be a frequency of $10.6 \%$. Moreover this frequency is the highest in case of samples received from newborn patients. The critical value frequency for creatinine was observed to be $2.9 \%$ as compared to $5.9 \%$ in case of adults. It is also important to note that the frequency of critical value notification is $0.9 \%$ in newborns which is lesser than in adults (7.1\%) as can be observed in table 2. Additionally, it was also seen in the present study that the frequency of critical value notification was $1.9 \%$ and 2.9 $\%$ in case of glucose and protein in cerebrospinal fluid analysis (CSF glucose and CSF protein) as can be seen in table 2.

\section{DISCUSSION}

The current study comprised of total 183056 tests which were analysed for three months during the period from July 2019 to September 2019 at emergency biochemistry laboratory at Safdarjung Hospital, New Delhi, India. As per current literature, studies from other laboratories had sample size ranging from 548786 done by Sarita et al to 5105336 studied by Anand Dighe ${ }^{10}$ et al. Moreover, it is evident from our study that the incidence of critical values in our lab varies from as low as $0.8 \%$ for glucose (children) to as high as 10.6 $\%$ for serum potassium in children, as depicted in table 2 . It was seen that the frequency of critical value notification for serum creatinine was $5.9 \%$ and it was $3.4 \%$ for blood Urea. In previous studies, Sarita et al have reported that the critical values frequencies of creatinine and urea was observed to be $6.59 \%$ and $28.78 \%$ respectively. Thus, there is a wide range of this frequency. As is depicted by this data, the frequency of critical values of renal function tests are much lower in our laboratory.

Additionally, it is evident from the present comparative study that the critical values of electrolytes are lower than study of Sarita et al but it is much higher than study done by A. Dighe and AA-Rocha et al. ${ }^{9}$ Also, it was also observed that incidence of critical values in serum potassium was more in paediatric patients as compared to adults. This finding could be attributed to the fact that there is more risk and incidence of haemolysis while drawing sample in children and newborn. This is because it is difficult to draw blood in paediatric patients. Moreover, these results also point towards the need of further studies in emergency laboratories to see the prevalence of critical values of serum electrolyte parameters in these patients to observe a pattern of serum electrolyte values in paediatric patients. Also, the frequencies of notification of critical values in the case of Serum total bilirubin was $10.4 \%$ in our study and it was 8.54 $\%$ in the study done by Sarita et al. Thus, in the case of total bilirubin also there is a wide variation among different laboratories.

Thus, this study highlights the need for analysis of frequency of critical values in emergency laboratories to identify the parameters with maximum critical values and also recognize the underlying causes for the same and take suitable measures, if necessary. Moreover, it is also important for laboratories to individualize the parameters for critical value notification in their respective laboratories and also set the upper and lower limits in consultation with the clinicians. Moreover, it is also imperative to train all laboratory staff including technicians and doctors on duty for the protocol of the laboratory regarding critical value notification and record the same in the respective registers. This is because this small step towards patient care can help modify clinical management of the patients and thus can be very useful for both clinicians and patients. Moreover, this will also in establishing a healthy interaction with the clinicians and laboratory specialist which will be beneficial in the long run. 


\section{CONCLUSIONS}

The frequency of critical value for serum potassium, is highest followed by serum sodium, in case of samples received from adult patients. Also, frequency of critical values for serum potassium in paediatric patients is even higher than adults. These values show that these critical biochemical values can be detrimental to these patients and timely notification to the clinician is very important so that necessary actions could be taken to correct them. Further studies of critical values in emergency labs is required to observe trends and identify the underlying causes and suggest suitable remedial measures if any.

\section{REFERENCES}

[1] College of American Pathologists. Laboratory Accreditation checklist. http://www.cap.org/apps/cap.portal.

[2] Lundberg GD. When to panic over abnormal values. Med Lab Observer 1972;4:47-54.

[3] Joint Commission on Accreditation of Healthcare Organizations. International Patient Safety Goals, 2007.
[4] Genzen JR, Tormey CA, Education Committee of the Academy of Clinical Laboratory Physicians and Scientists. Pathology consultation on reporting of critical values. Am J Clin Pathol 2011;135(4):505-13.

[5] Emancipator K. Critical values: ASCP practice parameter. American Society of Clinical Pathologists. Am J Clin Pathol 1997;108(3):247-53.

[6] Burtis C, Ashwood E, Bruns D. Tietz Textbook of Clinical Chemistry and Molecular Diagnostics. $4^{\text {th }}$ edn. St. Louis, MO: Elsevier Saunders 2006.

[7] Howanitz PJ, Steindel SJ, Heard NV. Laboratory critical values policies and procedures: a College of American Pathologists Q-Probes study in 623 institutions. Arch Pathol Lab Med 2002;126(6):663-9.

[8] Kuperman GJ, Boyle D, Jha A, et al. How promptly are inpatients treated for critical laboratory results? J Am Med Inform Assoc 1998;5(1):112-9.

[9] Arbiol-Roca A, Corral-Comesana S, Cano-Corres R, et al. Analysis of laboratory critical values at a referral Spanish tertiary university hospital. Biochem Med (Zagreb) 2019;29(1):010704.

[10] Dighe AS, Rao A, Coakley AB, et al. Analysis of laboratory critical value reporting at a large academic medical center. Am J Clin Pathol 2006;125(5):758-64. 